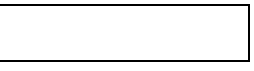

\author{
Military Technical College \\ Kobry El-Kobbah, \\ Cairo, Egypt
}

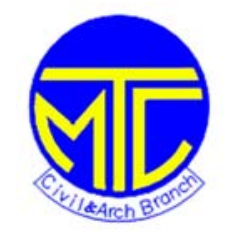

\section{$8^{\text {th }}$ International Conference on Civil and Architecture \\ Engineering \\ ICCAE-8-2010}

\title{
Convolutional-PML for Non-linear Analysis of Soil-Structure Interaction
}

\author{
By
}

\author{
Hiroo SHIOJIRI* \\ Laheman PERHAT $^{* *}$ \\ Zu-han $\mathrm{LI}^{* *}$
}

\section{$\underline{\text { Abstract: }}$}

Soil-structure interaction has significant effect on the dynamic responses of structures. In this paper, a time domain implicit convolutional-PML for structurefoundation soil system is developed for FEM analysis to absorb outgoing waves at the artificial boundaries of numerical models. At first, convolutional PML for FE analysis was developed, and symmetric coefficient matrices are obtained to be consistent with most of FEM codes. Then, the convolutional PML was extended to cope with elastoplastic material. The high performances of the proposed procedures both for linearelastic soil-structure problem and for non-linear soil-structure problem with elastoplastic soil subjected to large seismic loading are demonstrated by numerical examples.

\section{Keywords:}

Convolutional PML, Soil-structire-interaction, Finite element method

* College of Science and Technology,Nihon University,Japan

** Graduate School of College of Science and Technology,Nihon University,Japan 
Proceedings of the $\mathbf{8}^{\text {th }}$ ICCAE-8 Conference, 25-27 May, 2010

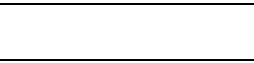

\section{Introduction:}

Demand for a nonlinear analysis has increased by introduction of a performance design of structures, a rapid progress of capability of computers, etc.. Nonlinear analysis usually requires far more amount of computation than linear analysis. To reduce the computation time in the nonlinear analysis, further development of computational procedures and computer capability is essential. In this study, an efficient method for the nonlinear analysis of the semi-infinite foundation was proposed. Several methods have been used in the nonlinear analysis of semi-infinite foundation soil [1]. The first is the extensive mesh models using a finite element method or a finite difference method with approximate energy transmitting boundaries. The second is the substructure method using, for example, finite element and time domain boundary element method. In the former, the degrees of freedom of the models are often very large. The latter method may be more efficient, but the nonlinearity must be restricted within the nearby portion of structures modeled by finite elements. The third is FEM with PML or convoltional PML. PML and convolutional-PML are proved to have efficient wave absorbing capability for linear elasto-dynamic problem, and the nonlinearity must be restricted within finite element domain. In the severe earthquakes, soil may become nonlinear to a large extent so that the second and the third methods will be inadequate.In this paper, convolutional-PML is extended to cope with non-linear problem, so that nonlinear soil can be analyzed with a limited number of meshes without loss of accuracy. This method is an extension of the method for a linear soil which maps an infinite domain into a finite domain, as described in [2][3][4][5].

\section{Methods:}

\subsection{Convolutional PML for nonlinear finite element analysis}

we introduce complex coordinate stretching function in frequency domain analysis as: $\tilde{\mathrm{x}}_{\mathrm{i}}=\int_{0}^{\mathrm{x}_{\mathrm{i}}} \lambda_{\mathrm{i}}(\mathrm{s}) \mathrm{ds}$

, where $x_{i}$ denotes $i$ th coordinate, and $\tilde{x}_{i}$ the corresponding transformed coordinate, and $\lambda_{i}$ is given as:

$$
\lambda_{\mathrm{i}}=\mathrm{k}_{\mathrm{i}}+\frac{\sigma_{\mathrm{i}}}{\alpha_{\mathrm{i}}+\mathrm{i} \omega}
$$

, where i is pure imaginary number, $\omega$ circular frequency, and $\mathrm{k}_{\mathrm{i}}, \alpha_{\mathrm{i}}$ and $\sigma_{\mathrm{i}}$ are non-negative continuous functions, such that $\mathrm{k}_{\mathrm{i}}=1$, and $\sigma_{\mathrm{i}}=1$ at FEM-PML interface.

At first, equations for elastic wave are formulated in $\tilde{\mathrm{x}}_{\mathrm{i}}$ coordinate, and then transformed to $\mathrm{x}_{\mathrm{i}}$ coordinate. Equations of motion are given as: 
Proceedings of the $\mathbf{8}^{\text {th }}$ ICCAE-8 Conference, 25-27 May, 2010

$-\omega^{2} \rho \bar{u}_{i}=\frac{1}{\lambda_{j}} \frac{\partial \bar{\sigma}_{i j}}{\partial x_{j}}+\rho g_{i} \delta(\omega)$

,where $\rho$ is density, $x_{j}$ is $j$ th coordinate, $\bar{u}_{i}$ is ith component of displacement, $\delta(\omega)$ is Derac's

Delta function, and $\mathrm{g}_{\mathrm{i}}$ is ith component of body force. In two-dimensional case, it is written as :

$$
\begin{array}{r}
-\rho \omega^{2}\left\{\mathrm{k}_{1} \mathrm{k}_{2}+\frac{\mathrm{k}_{2} \sigma_{1}\left(\alpha_{2}-\alpha_{1}\right)+\sigma_{1} \sigma_{2}}{\left(\alpha_{2}-\alpha_{1}\right)\left(\alpha_{1}+i \omega\right)}+\frac{\mathrm{k}_{1} \sigma_{2}\left(\alpha_{2}-\alpha_{1}\right)-\sigma_{1} \sigma_{2}}{\left(\alpha_{2}-\alpha_{1}\right)\left(\alpha_{2}+i \omega\right)}\right\} \overline{\mathrm{u}}_{i}=\frac{\partial}{\partial \mathrm{x}_{\mathrm{j}}}\left\{\left(\mathrm{k}_{\dot{j}}+\frac{\sigma_{\dot{j}}}{\alpha_{\dot{f}}+\mathrm{i} \omega}\right) \frac{\partial \bar{\sigma}_{\mathrm{ij}}}{\partial \mathrm{x}_{\mathrm{j}}}\right\} \\
+\rho\left\{\mathrm{k}_{1} \mathrm{k}_{2}+\frac{\mathrm{k}_{2} \sigma_{1}\left(\alpha_{2}-\alpha_{1}\right)+\sigma_{1} \sigma_{2}}{\left(\alpha_{2}-\alpha_{1}\right)\left(\alpha_{1}+i \omega\right)}+\frac{\mathrm{k}_{1} \sigma_{2}\left(\alpha_{2}-\alpha_{1}\right)-\sigma_{1} \sigma_{2}}{\left(\alpha_{2}-\alpha_{1}\right)\left(\alpha_{2}+i \omega\right)}\right\} \mathrm{g}_{\mathrm{i}} \delta(\omega)
\end{array}
$$

,where $\mathrm{j}$ indicates other index than $\mathrm{j}$.

For strain,we get:

$\overline{\mathrm{f}}_{\mathrm{ij}}=\frac{1}{\mathrm{j}} \frac{\partial \overline{\mathrm{u}}_{\mathrm{i}}}{\partial \mathrm{x}_{\mathrm{j}}}$

$\bar{\varepsilon}_{\mathrm{ij}}=\frac{1}{2}\left(\overline{\mathrm{f}}_{\mathrm{ij}}+\overline{\mathrm{f}}_{\mathrm{ji}}\right)$

Weak form equations with weight function $\mathrm{w}_{\mathrm{i}}$ are given as:

$$
\begin{aligned}
& \int_{\mathrm{v}}-\rho \omega^{2} \mathrm{w}_{\mathrm{i}}\left\{\mathrm{k}_{1} \mathrm{k}_{2}+\frac{\mathrm{k}_{2} \sigma_{1}\left(\alpha_{2}-\alpha_{1}\right)+\sigma_{1} \sigma_{2}}{\left(\alpha_{2}-\alpha_{1}\right)\left(\alpha_{1}+\mathrm{i} \omega\right)}+\frac{\mathrm{k}_{1} \sigma_{2}\left(\alpha_{2}-\alpha_{1}\right)-\sigma_{1} \sigma_{2}}{\left(\alpha_{2}-\alpha_{1}\right)\left(\alpha_{2}+i \omega\right)}\right\} \overline{\mathrm{u}}_{\mathrm{i}} \mathrm{dv} \\
& =\int \mathrm{w}_{\mathrm{i}}\left(\mathrm{k}_{\dot{f}}+\frac{\sigma_{\dot{j}}}{\alpha_{\dot{j}}+\mathrm{i} \omega}\right) \bar{\sigma}_{\mathrm{ij}} \mathrm{n}_{\mathrm{j}} \mathrm{ds}-\int \frac{\partial \mathrm{w}_{\mathrm{i}}}{\partial \mathrm{x}_{\mathrm{j}}}\left(\mathrm{k}_{\dot{j}}+\frac{\sigma_{\dot{j}}}{\alpha_{\dot{j}}+\mathrm{i} \omega}\right) \bar{\sigma}_{\mathrm{ij}} \mathrm{du} \\
& +\int_{\mathrm{v}} \rho \mathrm{w}_{\mathrm{i}}\left\{\mathrm{k}_{1} \mathrm{k}_{2}+\frac{\mathrm{k}_{2} \sigma_{1}\left(\alpha_{2}-\alpha_{1}\right)+\sigma_{1} \sigma_{2}}{\left(\alpha_{2}-\alpha_{1}\right)\left(\alpha_{1}+\mathrm{i} \omega\right)}+\frac{\mathrm{k}_{1} \sigma_{2}\left(\alpha_{2}-\alpha_{1}\right)-\sigma_{1} \sigma_{2}}{\left(\alpha_{2}-\alpha_{1}\right)\left(\alpha_{2}+\mathrm{i} \omega\right)}\right\} \mathrm{g}_{\mathrm{i}} \delta(\omega) \mathrm{dv}
\end{aligned}
$$

Applying inverse Fourier transform, the following equations in time domain are obtained:

$$
\begin{array}{r}
\int_{v} \rho w_{i}\left\{k_{1} k_{2} \ddot{u}_{i}+\frac{k_{j} \sigma_{j}\left(\alpha_{j}-\alpha_{j}\right)+\sigma_{1} \sigma_{2}}{\alpha_{j}-\alpha_{j}} e^{-\alpha_{j} t} * \ddot{u}_{i}\right\} d v \\
=\int w_{i}\left(k_{j} \sigma_{i j}+\sigma_{j} e^{-\alpha_{j} t} * \sigma_{i j}\right) n_{j} d s-\int \frac{\partial w_{i}}{\partial x_{j}}\left(k_{j} \sigma_{i j}+\sigma_{j} e^{-a_{j} t} * \sigma_{i j}\right) d v \ldots .
\end{array}
$$

,where * indicates convolutional integration.

Similarly ,from Eq. 5,we get:

$$
\left(\mathrm{k}_{\mathrm{j}} \mathrm{f}_{\mathrm{ij}}+\sigma_{\mathrm{j}} \mathrm{e}^{-\alpha_{\mathrm{i}} \mathrm{t}} * \mathrm{f}_{\mathrm{ij}}\right)=\frac{\partial \mathrm{u}_{\mathrm{i}}}{\partial \mathrm{x}_{\mathrm{j}}}
$$


$\cong \Delta \mathrm{t}\left\{(1-\theta) \mathrm{e}^{-\alpha \Delta t} \mathrm{f}(\mathrm{t})+\theta \mathrm{f}(\mathrm{t}+\Delta \mathrm{t})\right\}+e^{-\alpha \Delta t} F(t)=\theta \Delta \mathrm{tf}(\mathrm{t}+\Delta \mathrm{t})+\mathrm{e}^{-\alpha \Delta t} \mathrm{~F}^{*}(\mathrm{t})$

,where $\Delta \mathrm{t}$ is time increment and:

$0 \leq \theta \leq 1, \mathrm{~F}_{(\mathrm{t})}^{*}=\mathrm{F}(\mathrm{t})+(1-\theta) \Delta \mathrm{tf}(\mathrm{t})$

From Eq.8,we obtain:

$\int_{v} \rho \mathrm{w}_{\mathrm{i}}\left\{\mathrm{k}_{1} \mathrm{k}_{2}+\theta \Delta \mathrm{t}\left(\mathrm{k}_{2} \sigma_{1}+\mathrm{k}_{1} \sigma_{2}\right)\right\} \ddot{\mathrm{u}}_{\mathrm{i}}(\mathrm{t}+\Delta \mathrm{t}) \mathrm{dv}+\int_{\mathrm{v}} \rho \mathrm{w}_{\mathrm{i}} \sum_{\mathrm{j}=1}^{2}\left\{\frac{\mathrm{k}_{\mathrm{j}} \sigma_{\mathrm{j}}\left(\alpha_{\mathrm{j}}-\alpha_{\mathrm{j}}\right)+\sigma_{1} \sigma_{2}}{\alpha_{\mathrm{j}}-\alpha_{\mathrm{j}}} \mathrm{e}^{-\alpha_{\mathrm{j}} \mathrm{t} t} \mathrm{U}_{\mathrm{ij}}^{*}(\mathrm{t})\right\} \mathrm{dv}$

$=\int \mathrm{w}_{\mathrm{i}}\left(\mathrm{k}_{\mathrm{j}} \sigma_{\mathrm{ij}}+\sigma_{\mathrm{j}} \mathrm{e}^{-\mathrm{a}_{\mathrm{f}}{ }^{\mathrm{f}} *} \sigma_{\mathrm{ij}} \mathrm{n}_{\mathrm{j}}\right) \mathrm{ds}+\int_{\mathrm{v}} \rho \mathrm{w}_{\mathrm{i}}\left\{\mathrm{k}_{1} \mathrm{k}_{2}+\theta \Delta \mathrm{t}\left(\mathrm{k}_{2} \sigma_{1}+\mathrm{k}_{1} \sigma_{2}\right)\right\} \mathrm{g}_{\mathrm{i}} \mathrm{dv}$

$+\int_{v} \rho w_{i} \sum_{j=1}^{2}\left\{\frac{k_{j} \sigma_{j}\left(\alpha_{j}-\alpha_{j}\right)+\sigma_{1} \sigma_{2}}{\alpha_{j}-\alpha_{j}} e^{-\alpha_{j} \Delta t} G_{i}^{*}\right\} d v-\int \frac{\partial w_{i}}{\partial x_{j}}\left\{\left(k_{j}+\theta \Delta t \sigma_{j}\right) \sigma_{i j}(t+\Delta t)+\sigma_{j} e^{-\alpha_{j} \Delta t} \Sigma_{i j}^{*}(t)\right\} d v$

,where :

$\mathrm{U}_{\mathrm{ij}}^{*}(\mathrm{t})=\int_{0}^{\mathrm{t}} \mathrm{e}^{-\alpha_{\mathrm{j}}\left(\mathrm{t} \mathrm{t}^{-}\right)} \ddot{\mathrm{u}}_{\mathrm{i}}\left(\mathrm{t}^{\prime}\right) \mathrm{dt} \mathrm{t}^{\prime}+\Delta \mathrm{t}(1-\theta) \ddot{\mathrm{u}}_{\mathrm{i}}(\mathrm{t}), \mathrm{G}_{\mathrm{ij}}^{*}(\mathrm{t})=\int_{0}^{\mathrm{t}} \mathrm{e}^{-\alpha_{\mathrm{j}}\left(\mathrm{t} \mathrm{t}^{\prime}\right)} \mathrm{g}_{\mathrm{i}} \mathrm{dt} \mathrm{t}^{\prime}+\Delta \mathrm{t}(1-\theta) \mathrm{g}_{\mathrm{i}}$,

$\Sigma_{i j}^{*}(t)=\int_{0}^{t} e^{-\alpha_{f}\left(t-t^{\prime}\right)} \sigma_{i j}\left(t^{\prime}\right) d t^{\prime}+\Delta t(1-\theta) \sigma_{i j}(t)$

Similarly,from Eq.9 we obtain:

$\Delta \mathrm{f}_{\mathrm{ij}}=\mathrm{f}_{\mathrm{ij}}(\mathrm{t}+\Delta \mathrm{t})-\mathrm{f}_{\mathrm{ij}}(\mathrm{t})=\frac{1}{\mathrm{k}_{\mathrm{j}}+\sigma_{\mathrm{j}} \theta \Delta \mathrm{t}} \frac{\partial \Delta \mathrm{u}_{\mathrm{i}}}{\partial \mathrm{x}_{\mathrm{j}}}-\frac{\sigma_{\mathrm{j}} \mathrm{e}^{-\mathrm{a}_{\mathrm{j}} \Delta \mathrm{t}}}{\mathrm{k}_{\mathrm{j}}+\sigma_{\mathrm{j}} \theta \Delta \mathrm{t}}\left(\mathrm{F}_{\mathrm{ij}}^{*}(\mathrm{t})-\mathrm{F}_{\mathrm{ij}}^{*}(\mathrm{t}-\Delta \mathrm{t})\right)$.

,where :

$F_{i j}^{*}(t)=\int_{0}^{t} e^{-a_{j}(t-t)} f_{i j}\left(t^{\prime}\right) d t+(1-\theta) \Delta t f_{i j}(t)$

Substitutig Eq.15 into Eq.12,we obtain:

$\int_{\mathrm{v}} \rho \mathrm{w}_{\mathrm{i}}\left\{\mathrm{k}_{1} \mathrm{k}_{2}+\theta \Delta \mathrm{t}\left(\mathrm{k}_{2} \sigma_{1}+\mathrm{k}_{1} \sigma_{2}\right)\right\} \ddot{\mathrm{u}}_{\mathrm{i}}(\mathrm{t}+\Delta \mathrm{t}) \mathrm{dv}+\int_{\mathrm{v}} \rho \mathrm{w}_{\mathrm{i}} \sum_{\mathrm{j}=1}^{2}\left\{\frac{\mathrm{k}_{\mathrm{j}} \sigma_{\mathrm{j}}\left(\alpha_{\mathrm{j}}-\alpha_{\mathrm{j}}\right)+\sigma_{1} \sigma_{2}}{\alpha_{\mathrm{j}}-\alpha_{\mathrm{j}}} \mathrm{e}^{-\alpha_{\mathrm{j}} \Delta t} \mathrm{U}_{\mathrm{ij}}^{*}(\mathrm{t})\right\} \mathrm{dv}$

$=\int \mathrm{w}_{\mathrm{i}}\left(\mathrm{k}_{\mathrm{j}} \sigma_{\mathrm{ij}}+\sigma_{\mathrm{j}} \mathrm{e}^{-a_{\mathrm{i}} \mathrm{t}} \sigma_{\mathrm{ij}} \mathrm{n}_{\mathrm{j}}\right) \mathrm{ds}+\int_{\mathrm{v}} \rho \mathrm{w}_{\mathrm{i}}\left\{\mathrm{k}_{1} \mathrm{k}_{2}+\theta \Delta \mathrm{t}\left(\mathrm{k}_{2} \sigma_{1}+\mathrm{k}_{1} \sigma_{2}\right)\right\} \mathrm{g}_{\mathrm{i}} \mathrm{dv}$

$+\int_{\mathrm{v}} \rho \mathrm{w}_{\mathrm{i}} \sum_{\mathrm{j}=1}^{2}\left\{\frac{\mathrm{k}_{\mathrm{f}} \sigma_{\mathrm{j}}\left(\alpha_{\mathrm{j}}-\alpha_{\mathrm{j}}\right)+\sigma_{1} \sigma_{2}}{\alpha_{\mathrm{j}}-\alpha_{\mathrm{j}}} \mathrm{e}^{-\alpha_{\mathrm{j}} \mathrm{At}} \mathrm{G}_{\mathrm{ij}}^{*}(\mathrm{t})\right\} \mathrm{dv}$

$+\int \frac{\partial \mathrm{w}_{\mathrm{i}}}{\partial \mathrm{x}_{\mathrm{j}}}\left[\left(\mathrm{k}_{\mathrm{j}}+\theta \Delta \mathrm{t} \sigma_{j}\right)\left\{\left(\partial \sigma_{\mathrm{ij}} / \partial \mathrm{f}_{\mathrm{kl}}\right)\left(\Delta \mathrm{f}_{\mathrm{kl}}\right)+\sigma_{\mathrm{ij}}(\mathrm{t})\right\}+\sigma_{j} \mathrm{e}^{-\mathrm{a}_{\mathrm{j}} \Delta t} \Sigma_{\mathrm{ij}}^{*}(\mathrm{t})\right] \mathrm{dv}$ 
$=\int \mathrm{w}_{\mathrm{i}}\left(\mathrm{k}_{\mathrm{f}} \sigma_{\mathrm{ij}}+\sigma_{\dot{j}} \mathrm{e}^{-a_{\mathrm{j}} \mathrm{t}} * \sigma_{\mathrm{ij}} \mathrm{n}_{\mathrm{j}}\right) \mathrm{ds}+\int_{\mathrm{v}} \rho \mathrm{w}_{\mathrm{i}}\left\{\mathrm{k}_{1} \mathrm{k}_{2}+\theta \Delta \mathrm{t}\left(\mathrm{k}_{2} \sigma_{1}+\mathrm{k}_{1} \sigma_{2}\right)\right\} \mathrm{g}_{\mathrm{i}} \mathrm{dv}$

$+\int_{\mathrm{v}} \rho \mathrm{w}_{\mathrm{i}} \sum_{\mathrm{j}=1}^{2}\left\{\frac{\mathrm{k}_{\mathrm{j}} \sigma_{\mathrm{j}}\left(\alpha_{\mathrm{j}}-\alpha_{\mathrm{j}}\right)+\sigma_{1} \sigma_{2}}{\alpha_{\mathrm{j}}-\alpha_{\mathrm{j}}} \mathrm{e}^{-\alpha_{\mathrm{j}} \mathrm{tt}} \mathrm{G}_{\mathrm{ij}}^{*}(\mathrm{t})\right\} \mathrm{dv}$

$-\int \frac{\partial \mathrm{w}_{\mathrm{i}}}{\partial \mathrm{x}_{\mathrm{j}}}\left[\frac{\mathrm{DI}\left(\partial \sigma_{\mathrm{ij}} / \partial \mathrm{f}_{\mathrm{kl}}\right)}{\left(\mathrm{k}_{\mathrm{j}}+\theta \Delta \mathrm{t} \sigma_{\mathrm{j}}\right)\left(\mathrm{k}_{1}+\sigma_{\mathrm{l}} \theta \Delta \mathrm{t}\right)}\left\{\frac{\partial \Delta \mathrm{u}_{\mathrm{k}}}{\partial \mathrm{x}_{1}}-\sigma_{1} \mathrm{e}^{-\alpha_{\Delta} \Delta t}\left(\Delta \mathrm{tf} \mathrm{f}_{\mathrm{kl}}(\mathrm{t})-\left(1-\mathrm{e}^{-\alpha_{\Delta} \Delta t}\right) \mathrm{F}_{\mathrm{kl}}^{*}(\mathrm{t}-\Delta \mathrm{t})\right)\right\}\right.$

$\left.+\left(\mathrm{k}_{\mathrm{j}}+\theta \Delta \mathrm{t} \sigma_{\mathrm{j}}\right) \sigma_{\mathrm{ij}}(\mathrm{t})+\sigma_{\mathrm{j}} \mathrm{e}^{-a_{\mathrm{j}} \Delta \mathrm{t}} \Sigma_{\mathrm{ij}}^{*}(\mathrm{t})\right] \mathrm{dv}$

Rearranging,we obtain:

$\int_{\mathrm{v}} \rho \mathrm{w}_{\mathrm{i}}\left\{\mathrm{k}_{1} \mathrm{k}_{2}+\theta \Delta \mathrm{t}\left(\mathrm{k}_{2} \sigma_{1}+\mathrm{k}_{1} \sigma_{2}\right)\right\} \ddot{\mathrm{u}}_{\mathrm{i}}(\mathrm{t}+\Delta \mathrm{t}) \mathrm{dv}+\int \frac{\mathrm{DI}\left(\partial \sigma_{\mathrm{ij}} / \partial \mathrm{f}_{\mathrm{kl}}\right)}{\mathrm{g}_{\mathrm{j}}^{\prime} \mathrm{g}_{\mathrm{l}}^{\prime}} \frac{\partial \mathrm{w}_{\mathrm{i}}}{\partial \mathrm{x}_{\mathrm{j}}} \frac{\partial \Delta \mathrm{u}_{\mathrm{k}}}{\partial \mathrm{x}_{1}} \mathrm{dv}$

$=\int \mathrm{w}_{\mathrm{i}}\left(\mathrm{k}_{\mathrm{j}} \sigma_{\mathrm{ij}}+\sigma_{\mathrm{j}} \mathrm{e}^{-a_{\mathrm{f}} \mathrm{t}} \boldsymbol{\sigma}_{\mathrm{j}_{\mathrm{ij}}} \mathrm{n}_{\mathrm{j}}\right) \mathrm{ds}+\int_{\mathrm{v}} \rho \mathrm{w}_{\mathrm{i}}\left\{\mathrm{k}_{1} \mathrm{k}_{2}+\theta \Delta \mathrm{t}\left(\mathrm{k}_{2} \sigma_{1}+\mathrm{k}_{1} \sigma_{2}\right)\right\} \mathrm{g}_{\mathrm{i}} \mathrm{dv}$

$+\int_{\mathrm{v}} \rho \mathrm{w}_{\mathrm{i}} \sum_{\mathrm{j}=1}^{2}\left\{\frac{\mathrm{k}_{\mathrm{j}} \sigma_{\mathrm{j}}\left(\alpha_{\mathrm{j}}-\alpha_{\mathrm{j}}\right)+\sigma_{1} \sigma_{2}}{\alpha_{\mathrm{j}}-\alpha_{\mathrm{j}}} \mathrm{e}^{-\alpha_{\mathrm{j}} \mathrm{tt}} \mathrm{G}_{\mathrm{ij}}^{*}(\mathrm{t})\right\} \mathrm{dv}$

$-\int_{v} \rho w_{i} \sum_{j=1}^{2}\left\{\frac{k_{j} \sigma_{j}\left(\alpha_{j}-\alpha_{j}\right)+\sigma_{1} \sigma_{2}}{\alpha_{j}-\alpha_{j}} e^{-\alpha_{j} \Delta t} U_{i j}^{*}(t)\right\} d v$

,where:

$\mathrm{g}_{\mathrm{j}}^{\prime}=\mathrm{k}_{\mathrm{j}}+\theta \Delta \mathrm{t} \sigma_{\mathrm{j}}, \mathrm{DI}=\mathrm{g}_{1}^{\prime} \mathrm{g}_{2}^{\prime}$

If $\left(\partial \sigma_{\mathrm{ij}} / \partial \mathrm{f}_{\mathrm{kl}}\right)$ is symmetric with respect to $(\mathrm{ij})$ and $(\mathrm{kl})$, then coefficient matrices of

PML equations are symmetric. For linear elastic case,we obtain:

$\int_{\bar{v}} \rho \mathrm{w}_{\mathrm{i}}\left\{\mathrm{k}_{1} \mathrm{k}_{2}+\theta \Delta \mathrm{t}\left(\mathrm{k}_{2} \sigma_{1}+\mathrm{k}_{1} \sigma_{2}\right)\right\} \mathrm{u}_{\mathrm{i}}(\mathrm{t}+\Delta \mathrm{t}) \mathrm{dv}+\int \frac{\partial \mathrm{w}_{\mathrm{i}}}{\partial \mathrm{x}_{\mathrm{j}}} \frac{\mathrm{DI} \cdot \mathrm{C}_{\mathrm{ijkl}}}{\mathrm{g}_{\mathrm{j}}^{\prime} \mathrm{g}_{\mathrm{l}}^{\prime}} \frac{\partial \mathrm{u}_{\mathrm{k}}}{\partial \mathrm{x}_{1}}(\mathrm{t}+\Delta \mathrm{t}) \mathrm{dv}$

$=\int \mathrm{w}_{\mathrm{i}}\left(\mathrm{k}_{\mathrm{j}} \sigma_{\mathrm{ij}}+\sigma_{\mathrm{j}} \mathrm{e}^{-\alpha_{\mathrm{i}} \mathrm{t} *} \sigma_{\mathrm{ij}} \mathrm{n}_{\mathrm{j}}\right) \mathrm{ds}+\int_{\mathrm{v}} \rho \mathrm{w}_{\mathrm{i}}\left\{\mathrm{k}_{1} \mathrm{k}_{2}+\theta \Delta \mathrm{t}\left(\mathrm{k}_{2} \sigma_{1}+\mathrm{k}_{1} \sigma_{2}\right)\right\} \mathrm{g}_{\mathrm{i}} \mathrm{dv}$

$+\int_{v} \rho w_{i} \sum_{j=1}^{2}\left\{\frac{k_{j} \sigma_{j}\left(\alpha_{j}-\alpha_{j}\right)+\sigma_{1} \sigma_{2}}{\alpha_{j}-\alpha_{j}} e^{-\alpha_{j} \Delta t} G_{i j}^{*}(t)\right\} d v-\int_{\bar{v}} \rho w_{i} \sum_{j=1}^{2}\left\{\frac{k_{j} \sigma_{j}\left(\alpha_{j}-\alpha_{j}\right)+\sigma_{1} \sigma_{2}}{\alpha_{j}-\alpha_{j}} e^{-\alpha_{j} \Delta t} U_{i j}^{*}(t)\right\} d v$

,where $C_{i j k l}$ is elasticity tensor.

If Newmark's integration scheme is adopted, that is, if it is assumed :

$\Delta \ddot{\mathrm{u}}_{\mathrm{i}}=\frac{1}{\beta \Delta \mathrm{t}^{2}} \Delta \mathrm{u}_{\mathrm{i}}-\frac{1}{\beta \Delta \mathrm{t}} \dot{\mathrm{u}}_{\mathrm{i}}-\frac{1}{2 \beta} \ddot{\mathrm{u}}_{\mathrm{i}}, \Delta \dot{\mathrm{u}}_{\mathrm{i}}=\frac{\gamma}{\beta \Delta \mathrm{t}} \Delta \mathrm{u}_{\mathrm{i}}-\frac{\gamma}{\beta} \dot{\mathrm{u}}_{\mathrm{i}}+\left(1-\frac{\gamma}{2 \beta}\right) \Delta \mathrm{t} \ddot{\mathrm{u}}_{\mathrm{i}}$ 
Then, we obtain :

$\int_{\mathrm{v}} \frac{1}{\beta \Delta \mathrm{t}^{2}} \rho \mathrm{w}_{\mathrm{i}}\left\{\mathrm{k}_{1} \mathrm{k}_{2}+\theta \Delta \mathrm{t}\left(\mathrm{k}_{2} \sigma_{1}+\mathrm{k}_{1} \sigma_{2}\right)\right\} \Delta \mathrm{u}_{\mathrm{i}} \mathrm{dv}+\int \frac{\partial \mathrm{w}_{\mathrm{i}}}{\partial \mathrm{x}_{\mathrm{j}}} \frac{\mathrm{DI} \cdot \mathrm{C}_{\mathrm{ijkl}}}{\mathrm{g}_{\mathrm{j}}^{\prime} \mathrm{g}_{1}^{\prime}} \frac{\partial \Delta \mathrm{u}_{\mathrm{k}}}{\partial \mathrm{x}_{1}} \mathrm{dv}$

$=\int \mathrm{w}_{\mathrm{i}}\left(\mathrm{k}_{\mathrm{j}} \sigma_{\mathrm{ij}}+\sigma_{\mathrm{j}} \mathrm{e}^{-\mathrm{a}_{\mathrm{f}} \mathrm{t}} * \sigma_{\mathrm{ij}} \mathrm{n}_{\mathrm{j}}\right) \mathrm{ds}+\int_{\mathrm{v}} \rho \mathrm{w}_{\mathrm{i}}\left\{\mathrm{k}_{1} \mathrm{k}_{2}+\theta \Delta \mathrm{t}\left(\mathrm{k}_{2} \sigma_{1}+\mathrm{k}_{1} \sigma_{2}\right)\right\} \mathrm{g}_{\mathrm{i}} \mathrm{dv}$

$+\int_{\mathrm{v}} \rho \mathrm{w}_{\mathrm{i}} \sum_{\mathrm{j}=1}^{2}\left\{\frac{\mathrm{k}_{\mathrm{j}} \sigma_{\mathrm{j}}\left(\alpha_{\mathrm{j}}-\alpha_{\mathrm{j}}\right)+\sigma_{1} \sigma_{2}}{\alpha_{j}-\alpha_{\mathrm{j}}} \mathrm{e}^{-\alpha_{\mathrm{j}} \Delta \mathrm{t}} \mathrm{G}_{\mathrm{ij}}^{*}(\mathrm{t})\right\} \mathrm{dv}-\left(1-\frac{1}{2 \beta}\right) \int_{\overline{\mathrm{v}}} \rho \mathrm{w}_{\mathrm{i}}\left\{\mathrm{k}_{1} \mathrm{k}_{2}+\theta \Delta \mathrm{t}\left(\mathrm{k}_{2} \sigma_{1}+\mathrm{k}_{1} \sigma_{2}\right)\right\} \ddot{\mathrm{u}}_{\mathrm{i}} \mathrm{dv}$

$-\int_{\overline{\mathrm{v}}} \frac{1}{\beta \Delta \mathrm{t}} \rho \mathrm{w}_{\mathrm{i}}\left\{\mathrm{k}_{1} \mathrm{k}_{2}+\theta \Delta \mathrm{t}\left(\mathrm{k}_{2} \sigma_{1}+\mathrm{k}_{1} \sigma_{2}\right)\right\} \dot{\mathrm{u}}_{\mathrm{i}} \mathrm{dv}-\int \frac{\partial \mathrm{w}_{\mathrm{i}}}{\partial \mathrm{x}_{\mathrm{j}}} \frac{\mathrm{DI} \cdot \mathrm{C}_{\mathrm{ijkl}}}{\mathrm{g}_{\mathrm{j}}^{\prime} \mathrm{g}_{1}^{\prime}} \frac{\partial \mathrm{u}_{\mathrm{k}}}{\partial \mathrm{x}_{1}} \mathrm{dv}$

$-\int_{\bar{v}} \rho w_{i} \sum_{j=1}^{2}\left\{\frac{k_{j} \sigma_{j}\left(\alpha_{\dot{j}}-\alpha_{j}\right)+\sigma_{1} \sigma_{2}}{\alpha_{\dot{j}}-\alpha_{j}} e^{-\alpha_{j} t} U_{i j}^{*}(t)\right\} d v$

$+\int \frac{\partial w_{i}}{\partial x_{j}} \frac{D I}{g_{j}^{\prime} g_{l}^{\prime}} C_{i j k l} e^{-\alpha_{l} \Delta t} \sigma_{l} F_{k l}^{*} d v-\int \frac{\partial w_{i}}{\partial x_{j}} \sigma_{j} e^{-\alpha_{j} \Delta t} \Sigma_{i j}^{*}(t) d v$

When Rayleigh's damping is assumed,eqations of motion are modified as:

$-\omega^{2} \rho \overline{\mathrm{u}}_{\mathrm{i}}+\mathrm{i} \omega \mathrm{A} \rho \overline{\mathrm{u}}_{\mathrm{i}}=\frac{1}{\lambda_{\mathrm{j}}} \frac{\partial \overline{\mathrm{\sigma}}_{\mathrm{ij}}}{\partial \mathrm{x}_{\mathrm{j}}}+\frac{1}{\lambda_{\mathrm{j}}} \mathrm{BC}_{\mathrm{ijkl}} \overline{\dot{\mathrm{f}}}_{\mathrm{kl}}+\rho \mathrm{g}_{\mathrm{i}} \delta(\omega)$.

,where A and B are Rayleigh's constants. Then ,we obtain:

$\int_{\mathrm{v}} \rho \mathrm{w}_{\mathrm{i}}\left\{\mathrm{k}_{1} \mathrm{k}_{2}+\theta \Delta \mathrm{t}\left(\mathrm{k}_{2} \sigma_{1}+\mathrm{k}_{1} \sigma_{2}\right)\right\} \ddot{\mathrm{u}}_{\mathrm{i}}(\mathrm{t}+\Delta \mathrm{t}) \mathrm{dv}+\mathrm{A} \int_{\mathrm{v}} \rho \mathrm{w}_{\mathrm{i}}\left\{\mathrm{k}_{1} \mathrm{k}_{2}+\theta \Delta \mathrm{t}\left(\mathrm{k}_{2} \sigma_{1}+\mathrm{k}_{1} \sigma_{2}\right)\right\} \dot{\mathrm{u}}_{\mathrm{i}}(\mathrm{t}+\Delta \mathrm{t}) \mathrm{dv}$

$+\mathrm{B} \int \frac{\boldsymbol{\Phi} \mathrm{w}_{\mathrm{i}}}{\boldsymbol{q}_{\mathrm{x}}} \frac{\mathrm{DI}}{\mathrm{g}_{\mathrm{j}}^{\prime} \mathrm{g}_{1}^{\prime}} \mathrm{C}_{\mathrm{ijk}} \frac{\partial \dot{\mathrm{u}}_{\mathrm{k}}}{\partial \mathrm{x}_{1}} \mathrm{dv}+\int \frac{\partial \mathrm{w}_{\mathrm{i}}}{\partial \mathrm{x}_{\mathrm{j}}} \frac{\mathrm{DI}\left(\partial \sigma_{\mathrm{ij}} / \partial \mathrm{f}_{\mathrm{kl}}\right)}{\mathrm{g}_{\mathrm{j}}^{\prime} \mathrm{g}_{\mathrm{l}}^{\prime}} \frac{\partial \Delta \mathrm{u}_{\mathrm{k}}}{\partial \mathrm{x}_{1}} \mathrm{dv}$

$=\int \mathrm{w}_{\mathrm{i}}\left(\mathrm{k}_{\mathrm{f}} \sigma_{\mathrm{ij}}+\sigma_{\mathrm{j}} \mathrm{e}^{-\mathrm{a}_{\mathrm{f}} \mathrm{t}} * \sigma_{\mathrm{ij}} \mathrm{n}_{\mathrm{j}}\right) \mathrm{ds}+\int_{\mathrm{v}} \rho \mathrm{w}_{\mathrm{i}}\left\{\mathrm{k}_{1} \mathrm{k}_{2}+\theta \Delta \mathrm{t}\left(\mathrm{k}_{2} \sigma_{1}+\mathrm{k}_{1} \sigma_{2}\right)\right\} \mathrm{g}_{\mathrm{i}} \mathrm{dv}$

$+\int_{v} \rho w_{i} \sum_{j=1}^{2}\left\{\frac{k_{j} \sigma_{j}\left(\alpha_{j}-\alpha_{j}\right)+\sigma_{1} \sigma_{2}}{\alpha_{j}-\alpha_{j}} e^{-\alpha_{j} \Delta t} G_{i j}^{*}(t)\right\} d v-\int_{v} \rho w_{i} \sum_{j=1}^{2}\left\{\frac{k_{j} \sigma_{j}\left(\alpha_{j}-\alpha_{j}\right)+\sigma_{1} \sigma_{2}}{\alpha_{j}-\alpha_{j}} e^{-\alpha_{j} \Delta t} U_{i j}^{*}(t)\right\} d v$

$-A \int_{v} \rho w_{i} \sum_{j=1}^{2}\left\{\frac{k_{j} \sigma_{j}\left(\alpha_{j}-\alpha_{j}\right)+\sigma_{1} \sigma_{2}}{\alpha_{j}-\alpha_{j}} e^{-\alpha_{j} \Delta t} \tilde{U}_{i j}^{*}(t)\right\} d v$

$+\int \frac{\partial \mathrm{w}_{\mathrm{i}}}{\partial \mathrm{x}_{\mathrm{j}}}\left[\frac{\mathrm{DI}\left(\partial \sigma_{\mathrm{ij}} / \partial \mathrm{f}_{\mathrm{kl}}\right)}{\mathrm{g}_{\mathrm{j}}^{\prime} \mathrm{g}_{\mathrm{l}}^{\prime}} \sigma_{\mathrm{l}} \mathrm{e}^{-\alpha_{\mathrm{i}} \mathrm{\Delta t}}\left(\mathrm{F}_{\mathrm{kl}}^{*}(\mathrm{t})-\mathrm{F}_{\mathrm{kl}}^{*}(\mathrm{t}-\Delta \mathrm{t})\right)-\left(\mathrm{k}_{\mathrm{j}}+\theta \Delta \mathrm{t} \sigma_{\mathrm{j}}\right) \sigma_{\mathrm{ij}}(\mathrm{t})-\sigma_{j} \mathrm{e}^{-\alpha_{\mathrm{j}} \Delta t} \Sigma_{\mathrm{ij}}^{*}(\mathrm{t})\right] \mathrm{dv}$

$+B \int \frac{\partial w_{i}}{\partial x_{j}}\left\{\frac{D I \sigma_{1} e^{-\alpha_{i} t}}{g_{j}^{\prime} g_{1}^{\prime}} C_{i j k l} \tilde{F}_{k l}(t)-\sigma_{j} e^{-a_{j} \Delta t} \tilde{\Sigma}_{i j}(t)\right\} d v$ 
Proceedings of the $\boldsymbol{8}^{\text {th }}$ ICCAE-8 Conference, 25-27 May, 2010

\section{$\underline{2.2 \text { Constitutive Equations }}$}

Elast-plastic material model is used in this paper. Let $\mathrm{f}$ be yielding function and $\mathrm{g}$ be plastic potential .Then constitutive equations are given as:

$\mathrm{d} \sigma_{\mathrm{ij}}=\mathrm{C}_{\mathrm{ijkl}}\left(\mathrm{d} \varepsilon_{\mathrm{kl}}-\mathrm{d} \varepsilon_{\mathrm{kl}}^{\mathrm{p}}\right)=\mathrm{C}_{\mathrm{ijkl}}\left(\delta_{\mathrm{km}} \delta_{\mathrm{ln}}-\mathrm{B}_{\mathrm{klmn}}\right) \mathrm{d} \varepsilon_{\mathrm{mn}}$

, where $\mathrm{d} \sigma_{\mathrm{ij}}, \mathrm{d} \varepsilon_{\mathrm{kl}}$ and $\mathrm{d} \varepsilon_{\mathrm{kl}}^{\mathrm{p}}$ are stress increment, strain increment and plastic strain increment, respectively, and $\mathrm{B}_{\mathrm{klmn}}$ is given as:

$\mathrm{B}_{\mathrm{klmn}}=\frac{\partial \mathrm{f}}{\partial \sigma_{\mathrm{pq}}} \mathrm{C}_{\mathrm{pqmn}} \frac{\partial \mathrm{g}}{\partial \sigma_{\mathrm{kl}}} /\left(-\frac{\partial \mathrm{f}}{\partial \varepsilon_{\mathrm{ij}}^{\mathrm{p}}} \frac{\partial \mathrm{g}}{\partial \sigma_{\mathrm{ij}}}+\frac{\partial \mathrm{f}}{\partial \sigma_{\mathrm{ij}}} \mathrm{C}_{\mathrm{ijk}} \frac{\partial \mathrm{g}}{\partial \sigma_{\mathrm{kl}}}\right)$

For yielding function, we use Mohr-Coulomb function such as:

$\mathrm{f}\left(\mathrm{I}_{1}, \mathrm{~J}_{2}, \mathrm{~J}_{3}\right)=\frac{1}{2}\{3(1-\sin \phi) \sin \theta+\sqrt{3}(3+\sin \phi) \cos \theta\} \sqrt{\mathrm{J}_{2}}-\mathrm{I}_{1} \sin \phi-3 \cos \phi=0$

, where $I_{1}$ is the first scalar invariant of stress, $\mathrm{J}_{2}, \mathrm{~J}_{3}$ are the second and the third scalar invariants of stress deviator, respectively, $\phi$ is friction angle ,c is cohesive force and $\theta$ is given as:

$\theta=\frac{1}{3} \cos ^{-1}\left(-3 \sqrt{3} / 2 \times \mathrm{J}_{3} / \mathrm{J}_{2}^{3 / 2}\right) \quad(0<\theta<\pi / 3)$

For plastic potential ,Drucker Praeger function is used:

$\mathrm{g}\left(\mathrm{I}_{1}, \mathrm{~J}_{2}\right)=\sqrt{\mathrm{J}_{2}}-\alpha \mathrm{I}_{1} \quad(\alpha>0)$

For plane strain state, $\alpha$ is given as:

$\alpha=\tan \psi / \sqrt{9+12 \tan ^{2} \psi}$

,where $\psi$ is dilatational angle.

\section{Numerical example}

\subsection{One-dimensional model}

We consider one dimensional rod shown in Figure 1.

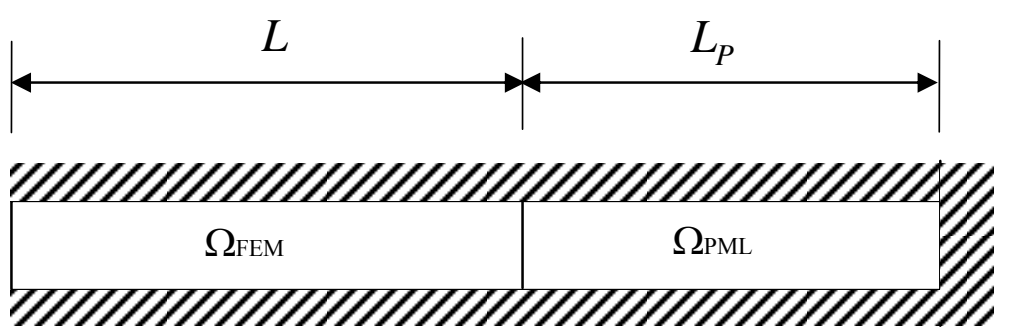

Figure (1): One-dimensional Model 
The stress-strain relationship is assumed to be elastoplastic.

Parameters are assumed as(all non-dimensional values): $\mathrm{c}=20, \phi=\psi=1.5$,Lame's constants $=12.5, \alpha_{1}=\left(\mathrm{x} / \mathrm{L}_{\mathrm{P}}\right) \alpha_{\mathrm{MAX}}, \quad \sigma_{1}=\left(\mathrm{x} / \mathrm{L}_{\mathrm{P}}\right) \sigma_{\mathrm{MAX}}, \quad \kappa_{1}=1+\left(\mathrm{x} / \mathrm{L}_{\mathrm{P}}\right) \kappa_{\mathrm{MAX}}, \quad \alpha_{\mathrm{MAX}}=1, \quad \sigma_{\mathrm{MAX}}=10, \quad$ an $d \kappa_{\mathrm{MAX}}=10$, where origin of $x$ is set at PML-FEM interface. We impose displacement at the left end of the rod. The displacement is time-harmonic with amplitude $30 \sqrt{\pi}$,. Mesh length is $1, L=100, L_{P}=20, \rho=1$, Time histories at $x=0$ are shown in Figure 2 and Figure 3 along with those obtained with viscous dashpots set at right end. 'Reference' in the figures indicates results of very long rod where reflected wave cannnot come back within time 60 .

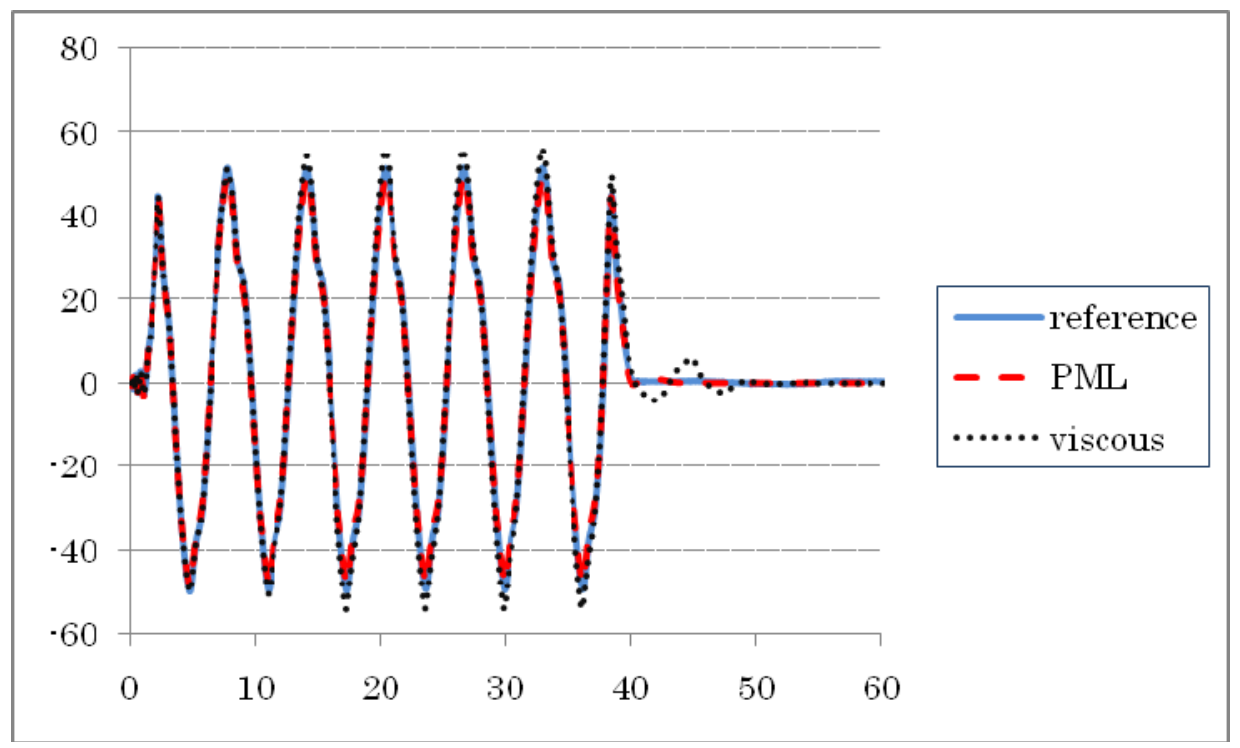

Figure (2):Velocity response

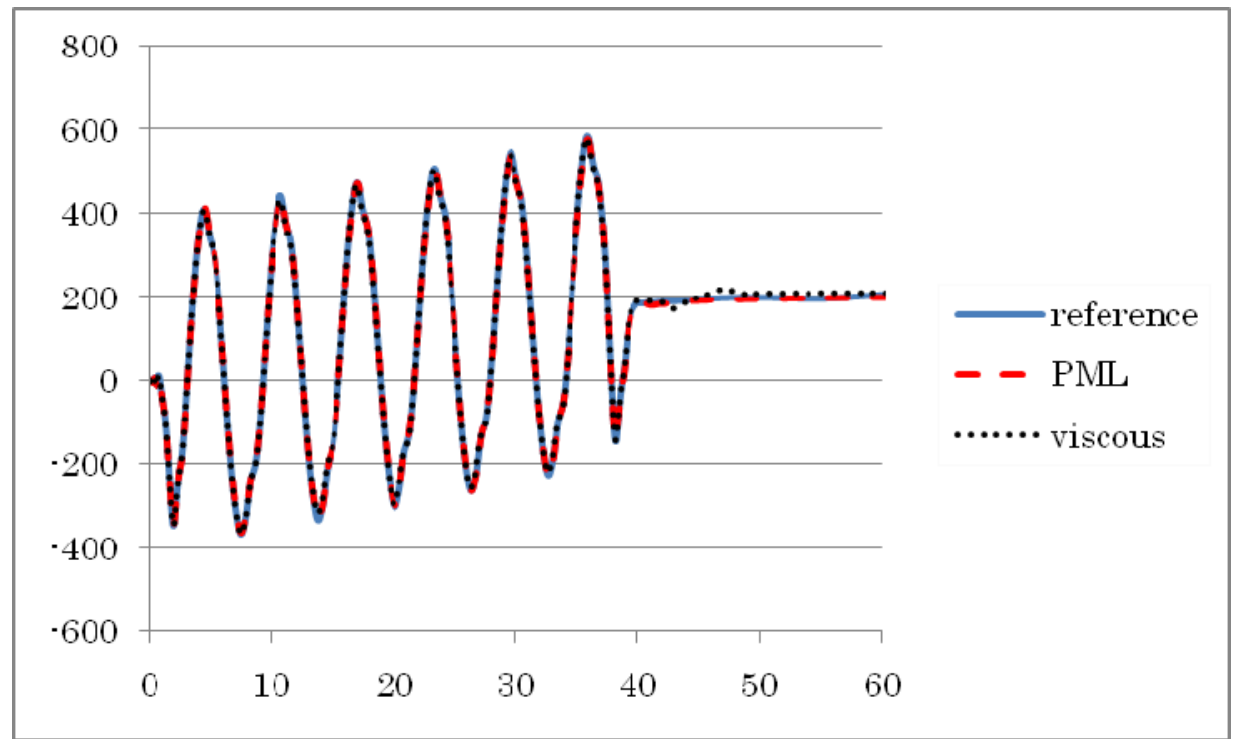

Figure (3):Strain response 
Proceedings of the $\mathbf{8}^{\text {th }}$ ICCAE-8 Conference, 25-27 May, 2010

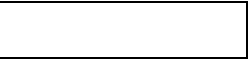

Reflected wave is much smaller with PML than with viscous dashpots in nonlinear case.

\subsection{Two-dimensional model}

Simple soil-structure interaction model is used as shown inFig.4. Rigid building with nondimensional height 4 and width 2 is placed on soil. Soil is of two layer. Top layer is elastoplastic with the same material properties as one-dimensional model, and thickness is 8(nondimensional-length). Bottom layer is elastic and of the same density and Lame's parameter with one dimensional model , and thickness is 20. Incident wave of Ricker's Wavelet with amplitude 50 is applied at the depth of 20 . Larger soil model has width 18 , while smaller soil model width 10. PML has thickness 5. PML parameters are also the same as one dimensional model. The results of 4 kindes of models are compared: first with larger soil and linear PML, second with smaller soil and linear PML,third with larger soil and non-linear PML,and last with smaller soil and non-linear PML. Responses at the top of building,those at depth 8 and 16 are shown in Fig,5 -8.

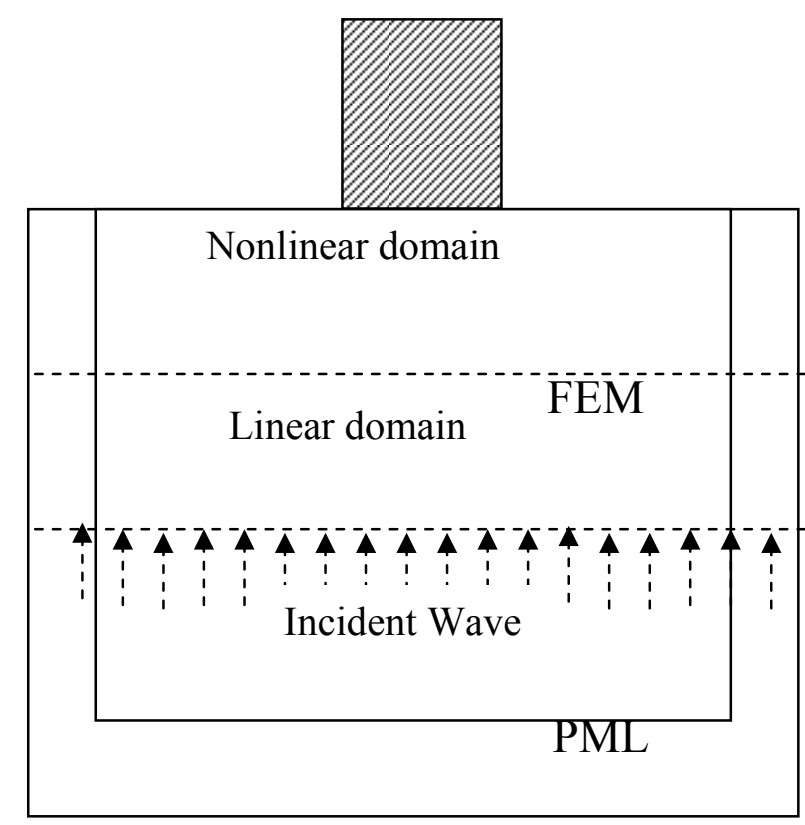

Figure (4):Soil-structure interaction Model

There are discrepancies between the results of larger and smaller model with linear PML, while results of larger and smaller models with non-linear PML coincide well. It indicate that we can use smaller soil domain when we use non-linear PMLand save computational time. 
Proceedings of the $\mathbf{8}^{\text {th }}$ ICCAE-8 Conference, 25-27 May, 2010

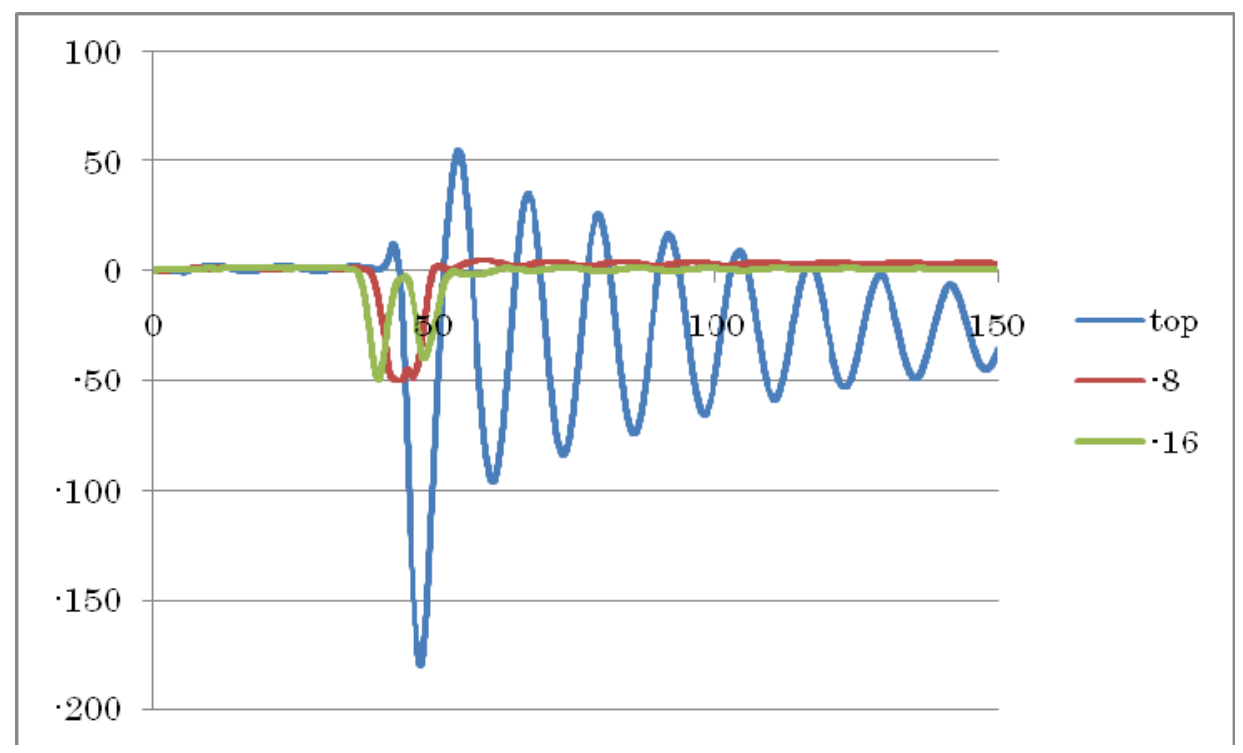

Figure (5):Smaller model with linear PML

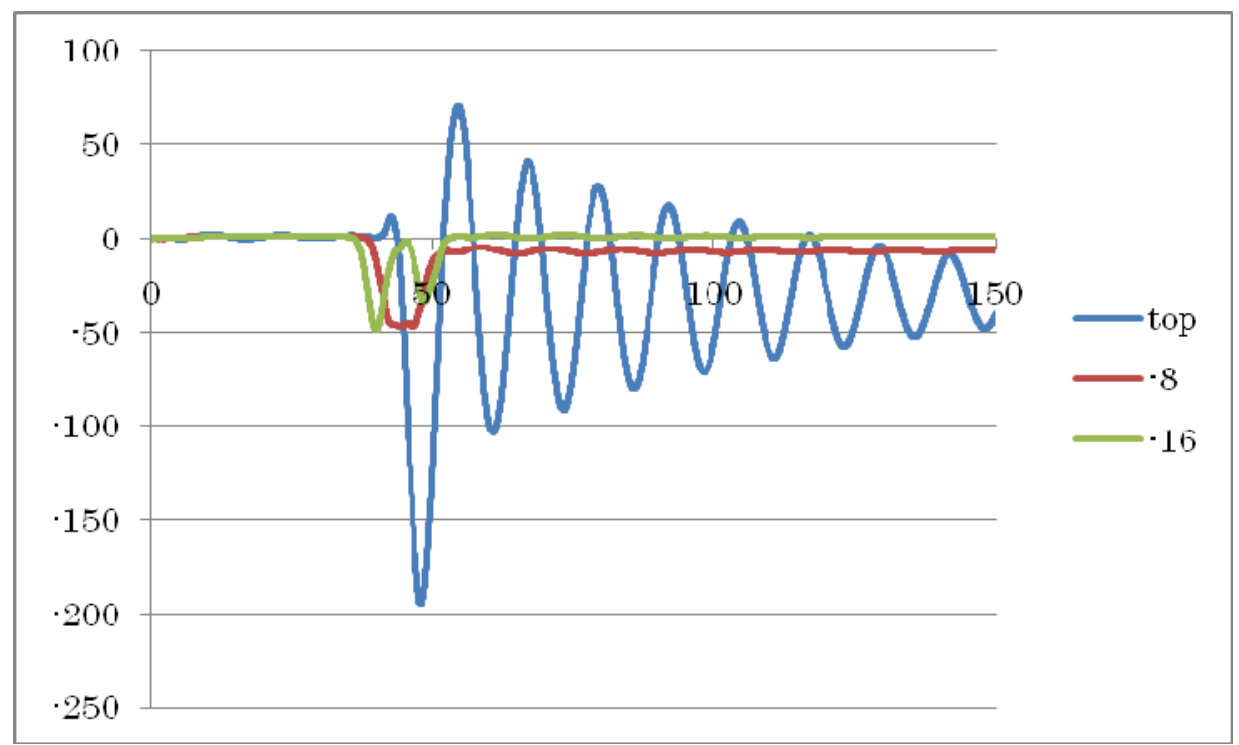

Figure (6):Larger er model with linear PML 
Proceedings of the $\mathbf{8}^{\text {th }}$ ICCAE-8 Conference, 25-27 May, 2010

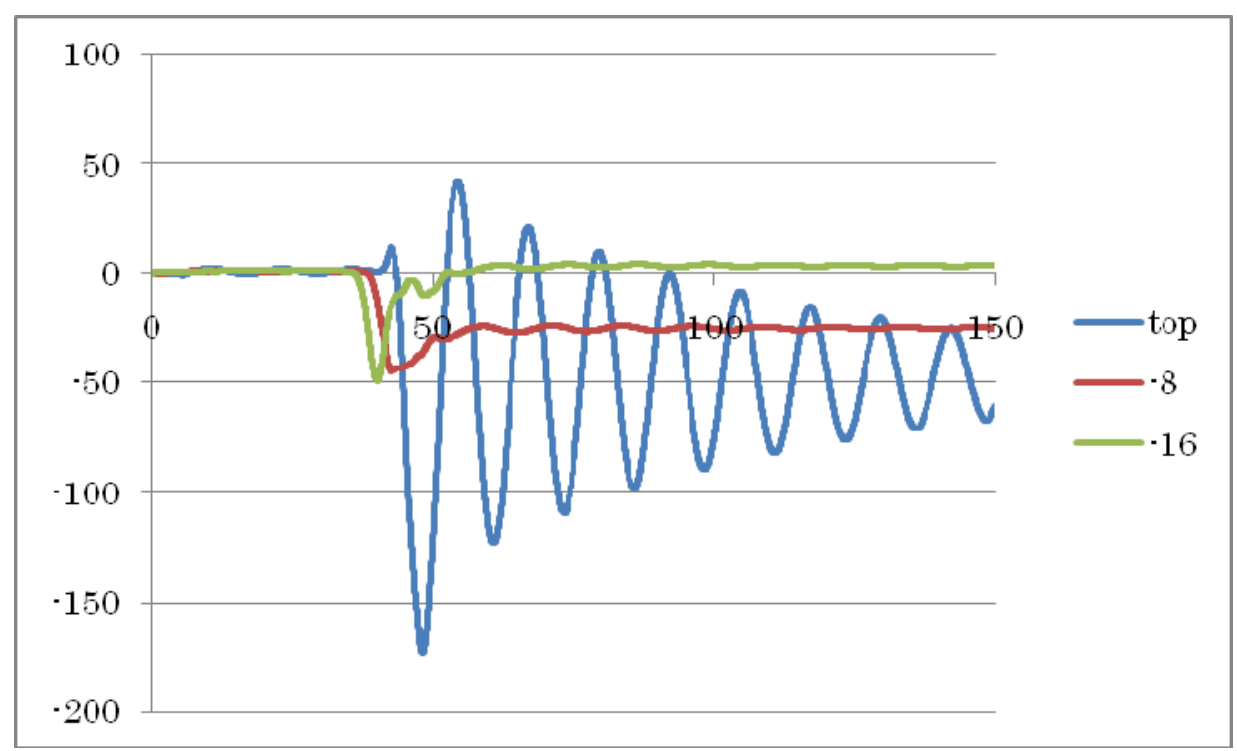

Figure (7):Smaller model with non-linear PML

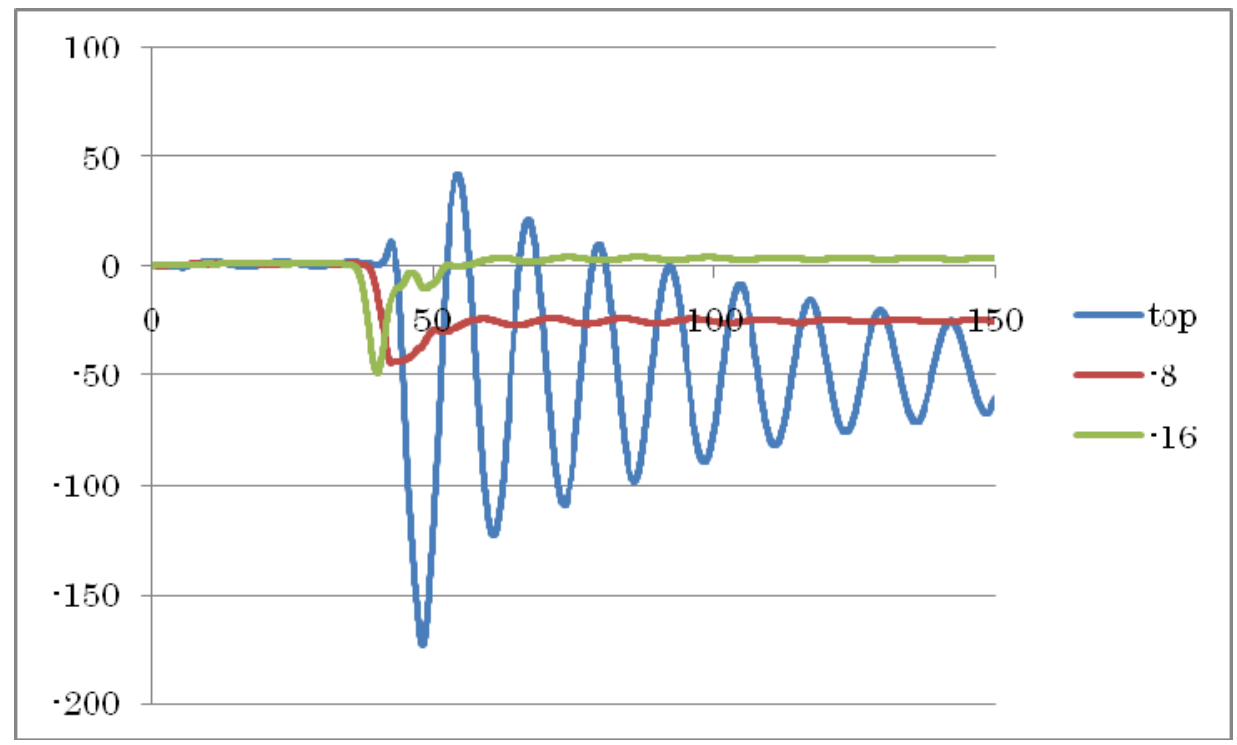

Figure (8):Larger model with non-linear PML 
Proceedings of the $\boldsymbol{8}^{\text {th }}$ ICCAE-8 Conference, 25-27 May, 2010

\section{Conclusions:}

Efficient displacement based convolutional PML procedures have been developed for application to non-linear dynamic response analyses of semi-infinite soil-structure interaction problems in time domain. It is demonstrated by numerical examples that the accuracy of the developed procedures is significantly better than that of viscous boundaries, and conventional linear PML.

\section{References:}

[1] J.P,Wolf, Soil-Structure-Interaction Analysis in time Domain, Prentice-Hall, Engelwood Cliffs, 446p, 1988.

[2] P.Berenger, A Perfectly Matched Layer for the Absorption of Electromagnetic Waves, Journal of Computational Physics, No.114, p.185-200, 1994.

[3] F. Collino, and C. Tsogka, Applicationof the perfectly matched absorbing layer to the linear elastodynamic problem in anisotropic heterogeneous media, Geophysics,vol. 66 ,No.1,p.294-307,2001

[4] U.Basu and A.K.Chopra: Perfectly matched layers for time-harmonic elestodynamics of unbounded domains: theory and finite-element implementation, Comput. Methods Appl. Mech. Eng.vol..192, p.1337-1375, 2003

[5] U.Basu and A.K.Chopra, Perfectly matched layers transient elestodynamics of unbounded domains, Int.J.Numerical Methods Eng.vol.59, p.1039-1074, 2004 